\title{
Sum rule approach to the isoscalar giant monopole resonance in drip line nuclei
}

\author{
M. Centelles, ${ }^{1}$ X. Viñas, ${ }^{1}$ S. K. Patra, ${ }^{2}$ J. N. De,${ }^{3,4}$ and Tapas Sil ${ }^{1}$ \\ ${ }^{1}$ Departament d'Estructura i Constituents de la Matèria, Facultat de Física, \\ Universitat de Barcelona, Diagonal 647, E-08028 Barcelona, Spain \\ ${ }^{2}$ Institute of Physics, Sachivalaya Marg, Bhubaneswar-751 005, India \\ ${ }^{3}$ Variable Energy Cyclotron Centre, 1/AF, Bidhannagar, Kolkata-700064, India \\ ${ }^{4}$ Saha Institute of Nuclear Physics, I/AF, Bidhannagar, Kolkata-700064, India
}

(Received 10 February 2005; published 14 July 2005)

\begin{abstract}
Using the density-dependent Hartree-Fock approximation and Skyrme forces together with the scaling method and constrained Hartree-Fock calculations, we obtain the average energies of the isoscalar giant monopole resonance. The calculations are done along several isotopic chains from the proton to the neutron drip lines. It is found that while approaching the neutron drip line, the scaled and the constrained energies decrease and the resonance width increases. Similar but smaller effects arise near the proton drip line, although only for the lighter isotopic chains. A qualitatively good agreement is found between our sum rule description and the presently existing random phase approximation results. The ability of the semiclassical approximations of the Thomas-Fermi type, which properly describe the average energy of the isoscalar giant monopole resonance for stable nuclei, to predict average properties for nuclei near the drip lines is also analyzed. We show that when $\hbar$ corrections are included, the semiclassical estimates reproduce, on average, the quantal excitation energies of the giant monopole resonance for nuclei with extreme isospin values.
\end{abstract}

DOI: 10.1103/PhysRevC.72.014304

PACS number(s): 24.30.Cz, 21.60.Jz, 21.10.Pc, 21.30.Fe

\section{INTRODUCTION}

Experimental and theoretical studies of exotic nuclei with extreme isospin values are presently one of the more active areas of research in nuclear physics. Recent developments in accelerator technology and detection techniques allow research beyond the limits of $\beta$ stability. The number of unstable nuclei for which masses have been measured is rapidly increasing [1], and this trend is expected to continue because of the use of radioactive beams [2-4]. In particular, the proton drip line has been approached as far as for $\mathrm{Pb}$ isotopes [5]. The neutron drip line has been reached to date for $Z \leqslant 8$ and is expected to be extended to $Z \leqslant 28$ in the present decade [6].

Collective phenomena are very useful tools to study the nuclear structure as well as to test the ability of the nuclear effective forces in describing such situations. The analysis of the small amplitude oscillations, i.e., the giant resonances, is of special relevance. In particular, it is very interesting to study the isoscalar giant monopole resonance (ISGMR) from where the incompressibility modulus of nuclear matter $\left(K_{\infty}\right)$ can be extracted [7,8]. The value of $K_{\infty}$ is an important ingredient not only for the description of finite nuclei but also for the study of heavy ion collisions, supernovae, and neutron stars.

The ISGMR for stable nuclei has been studied long ago from both experimental and theoretical standpoints [9]. The basic theory for the microscopic description of these collective motions is the random phase approximation (RPA) $[10,11]$. The RPA calculations allow one to obtain the strength distribution $S(E)$ which measures the response of a nucleus to an external perturbation. In the case of the giant resonances, $S(E)$ is usually concentrated in a rather narrow region of the energy spectrum, at least for heavy stable nuclei. Thus the knowledge of a few low energy-weighted moments of $S(E)$ (sum rules) can provide useful information on the average properties of the giant resonances as, for instance, the energy of the centroids and the resonance widths. The full RPA calculation can be avoided by using the so-called sum rule approach in which several selected odd moments of $S(E)$ are obtained by means of the properties of the ground state only [12] and thus used to evaluate these average properties. However, the full quantal calculation of the sum rules is still a complicated task. In some particular cases, it can be simplified by using the scaling method [12-14] to obtain the cubic energy-weighted moment or performing constrained Hartree-Fock (HF) calculations $[7,12,14,15]$, which allow one to compute the inverse energy-weighted moment.

Nuclei near the drip lines are characterized by the small energy of the last bound nucleons and by their large asymmetry $I=(N-Z) / A$; therefore, it is expected that their properties, in particular those of the collective excitations, may considerably differ from the corresponding properties of the stable nuclei. The theoretical analysis of the giant monopole resonances of some exotic nuclei has been worked out in the last few years. For instance, the RPA formalism together with Skyrme forces have been used to study the ISGMR of some isotopes of $A=100,110$, and 120 [16] and of some $\mathrm{Ca}$ isotopes $[17,18]$. These RPA calculations give us detailed information about the specific nuclei considered. The qualitative behavior of the ISGMR of other exotic nuclei may be inferred from these RPA results; however, a wider study of the properties of the collective modes near the drip lines is still lacking. To obtain global insight into the behavior of the ISGMR near the drip lines, we will study in this paper the aforementioned average properties of this collective excitation using the sum rule approach along different isotopic chains covering the whole periodic table. The scaling transformation of the density applied together with zero-range Skyrme forces 
allows us to express, for the monopole oscillations, the $m_{3}$ RPA sum rule (i.e., the cubic weighted moment of the strength distribution) by a simple and closed formula related to different contributions to the ground-state energy [12]. Spherical constrained HF calculations also allow us to obtain the inverse energy-weighted moment of the strength distribution (the RPA $m_{-1}$ sum rule) for the monopole oscillation in an easy way [12]. Although with the sum rule approach detailed information on the RPA strength cannot be obtained, this method can easily provide us with some useful information about the average energies and widths of the ISGMR of nuclei extending from the stability to the drip lines.

The shell structure of nuclei near the drip lines considerably differs from the structure of stable nuclei. However, it should be pointed out that, in principle, the average properties of the giant resonances are not appreciably influenced by the pairing correlations, at least not for medium and relatively heavy subshell closed nuclei when calculated with Skyrme forces [17,19]. Thus, for a fast estimate of some general trends of the average properties of the ISGMR, we will restrict ourselves to nonrelativistic Skyrme HF calculations using the uniform filling approximation, in which particles occupy the lowest single-particle orbits from the bottom of the potential.

Semiclassical methods like the Thomas-Fermi (TF) theory and its extensions (ETF) which include $\hbar$ corrections [20] have proven to be very helpful in dealing with nuclear properties of global character that vary smoothly with the number of particles $A$ and in regions where the shell corrections (quantal effects) are small as compared with the average value provided by the semiclassical calculations [21,22]. Reproduction of the binding energy from the celebrated Bethe-Weizsäcker mass formula [23] is the best-known example of this kind. Semiclassical ETF-type calculations of the average energies of some collective oscillations, in particular the breathing mode, also reproduce smoothly the quantal RPA values [20,24-27] for nuclei close to the stability line. This can be understood because the ISGMR is a collective oscillation whose average properties are, in general, rather insensitive to shell effects which are absent in the semiclassical TF-type approaches. In this paper, we also want to investigate the ability of the TF approach and its extensions to reproduce the smooth variation with $A$ of the average energies and widths of the ISGMR near the drip lines.

The paper is organized as follows: In Sec. II, we review the basic theory of the sum rule approach applied to obtain the average energy of the ISGMR using Skyrme forces. The behavior of these average energies along different chains of isotopes is discussed in Sec. III paying special attention to the case of Ca isotopes. In Sec. IV, we study the semiclassical TF and ETF descriptions of the ISGMR, particularly near the drip lines. Finally, the summary and conclusions are given in the last section.

\section{THEORY}

The response of the ground state of a nucleus to the action of a multipole moment, represented by the operator $Q$, is completely characterized by its associated strength function
$S(E)$ defined as [12]

$$
S(E)=\sum_{n}|\langle n|Q| 0\rangle|^{2} \delta\left(E_{n}-E\right),
$$

where $|0\rangle$ and $|n\rangle$ are the normalized ground and excited states, respectively, and $E_{n}$ are the excitation energies.

The moments of the strength function are defined as

$$
m_{k}=\int_{0}^{\infty} E^{k} S(E) d E
$$

where $k$ is an integer. The different moments fulfill the inequalities [12]

$$
\sqrt{\frac{m_{k+1}}{m_{k-1}}} \geqslant \frac{m_{k}}{m_{k-1}} \geqslant \sqrt{\frac{m_{k}}{m_{k-2}}}
$$

from where one can define average energies as follows:

$$
\bar{E}_{k}=\sqrt{\frac{m_{k}}{m_{k-2}}} \quad \text { and } \quad \tilde{E}_{k}=\frac{m_{k}}{m_{k-1}} .
$$

In addition, the square of the variance of the strength is defined as [12]

$$
\sigma^{2}=\frac{m_{2}}{m_{0}}-\left(\frac{m_{1}}{m_{0}}\right)^{2} \leqslant \frac{1}{4}\left(\frac{m_{3}}{m_{1}}-\frac{m_{1}}{m_{-1}}\right) .
$$

With the help of the completeness relation $\sum_{n}|n\rangle\langle n|=1$, it can be verified that for any positive odd integer $k$ the moments (2) can be evaluated as the expectation value in the ground state $|0\rangle$ of some commutators involving the Hamiltonian $H$ and the operator $Q$ (assumed to be a Hermitian and one-body operator). For instance,

$$
m_{1}=\frac{1}{2}\langle 0|[Q,[H, Q]]| 0\rangle,
$$

and

$$
m_{3}=\frac{1}{2}\langle 0|[[Q, H],[H,[H, Q]]]| 0\rangle,
$$

which are called the energy-weighted and cubic energyweighted sum rules, respectively [12]. Equations (6) and (7) as they are written are not useful for practical calculations because the exact ground state $|0\rangle$ is usually unknown. However, when the moments are computed within the $1 \mathrm{p} 1 \mathrm{~h}$ RPA it can be shown that they coincide exactly with the result obtained by replacing the actual ground state $|0\rangle$ by the uncorrelated HF wave function $|\mathrm{HF}\rangle[12,28]$.

As usual, to evaluate the energy-weighted sum rule $m_{1}$, we will restrict ourselves to an isoscalar single-particle operator $Q=\sum_{i} f\left(r_{i}\right)$ and neglect the momentum-dependent parts of the residual interaction. In this case one has contributions coming only from the kinetic energy, and at the RPA level one finds

$$
m_{1}=\frac{1}{2}\langle\mathrm{HF}|[Q,[H, Q]]| \mathrm{HF}\rangle=\frac{\hbar^{2}}{2 m}\left\langle\mathrm{HF}\left|(\nabla f)^{2}\right| \mathrm{HF}\right\rangle .
$$

It has been shown that Eq. (8) also holds for a Skyrme force because of the $\delta$ character of the $p^{2}$ terms [29]. For the isoscalar monopole oscillation, one has $Q=\sum_{i=1}^{A} r_{i}^{2}$, and thus the RPA $m_{1}$ sum rule becomes

$$
m_{1}=\frac{2 \hbar^{2}}{m} A\left\langle r^{2}\right\rangle
$$


where the expectation value of the operator $r^{2}$ is calculated with the HF wave function.

\section{A. The scaling approach with Skyrme forces}

As we know [12], the cubic energy-weighted moment $m_{3}$ of the RPA strength function can be calculated through the scaled ground-state wave function $\Phi_{\eta}$ defined as

$$
\left|\Phi_{\eta}\right\rangle=e^{-i \eta Q_{1}}\left|\Phi_{0}\right\rangle
$$

where $\eta$ is an arbitrary scaling parameter and $Q_{1}=i[H, Q]$, for a Hermitian one-body operator $Q$. Then,

$$
m_{3}=\frac{1}{2} \frac{\partial^{2}}{\partial \eta^{2}}\left[\left\langle\Phi_{\eta}|H| \Phi_{\eta}\right\rangle\right]_{\eta=0} .
$$

The $m_{3}$ moment measures the change of the energy of the nucleus when the ground-state wave function is deformed according to (10). If $Q$ is the monopole collective operator defined previously, the scaling transformation (10) induces a change of scale in the ground-state HF wave function $\Phi_{0}$ conserving its normalization, i.e., each single-particle wave function varies as

$$
\phi_{\eta}^{M}=e^{3 \tilde{\eta} / 2} \phi_{0}\left(e^{\tilde{\eta}} x, e^{\tilde{\eta}} y, e^{\tilde{\eta}} z\right),
$$

where $\tilde{\eta}=-2 \hbar^{2} \eta / m$, and $\phi_{0}$ are the single-particle wave functions comprised in $\Phi_{0}$.

Under the monopole transformation (12), the particle, kinetic, and spin densities that enter into the Skyrme energy density scale as

$$
\rho_{\lambda}(\boldsymbol{r})=\lambda^{3} \rho(\lambda \boldsymbol{r}), \quad \tau_{\lambda}(\boldsymbol{r})=\lambda^{5} \tau(\lambda \boldsymbol{r}), \quad \boldsymbol{J}_{\lambda}(\boldsymbol{r})=\lambda^{5} \boldsymbol{J}(\lambda \boldsymbol{r}),
$$

where $\lambda=e^{\tilde{\eta}}$. Inserting these scaled densities in the Skyrme energy density functional, the scaled energy is obtained as

$$
E(\lambda)=\lambda^{2} T+\lambda^{3} E_{\delta}+\lambda^{5}\left(E_{\mathrm{fin}}+E_{\mathrm{so}}\right)+\lambda^{3 \gamma+3} E_{\rho}+\lambda E_{C},
$$

where $T$ and $E_{C}$ are the kinetic and Coulomb energies and $E_{\delta}, E_{\mathrm{fin}}, E_{\mathrm{so}}$, and $E_{\rho}$ are the different contributions to the potential energy in the notation of Ref. [12]. They are given as

$$
\begin{aligned}
E_{\delta}=\int d \boldsymbol{r}\left\{\frac{1}{2} \rho^{2} t_{0}\left(1+\frac{x_{0}}{2}\right)-\frac{1}{2}\left(\rho_{n}^{2}+\rho_{p}^{2}\right) t_{0}\left(\frac{1}{2}+x_{0}\right)\right\}, \\
E_{\mathrm{fin}}=\int d \boldsymbol{r}\left\{\frac{1}{4} \rho \tau\left[t_{1}\left(1+\frac{x_{1}}{2}\right)+t_{2}\left(1+\frac{x_{2}}{2}\right)\right]\right. \\
-\frac{1}{4}\left(\rho_{n} \tau_{n}+\rho_{p} \tau_{p}\right)\left[t_{1}\left(\frac{1}{2}+x_{1}\right)-t_{2}\left(\frac{1}{2}+x_{2}\right)\right] \\
+\frac{1}{16}(\nabla \rho)^{2}\left[3 t_{1}\left(1+\frac{x_{1}}{2}\right)-t_{2}\left(1+\frac{x_{2}}{2}\right)\right] \\
-\frac{1}{16}\left[\left(\nabla \rho_{n}\right)^{2}+\left(\nabla \rho_{p}\right)^{2}\right]\left[3 t_{1}\left(\frac{1}{2}+x_{1}\right)\right. \\
\left.\left.+t_{2}\left(\frac{1}{2}+x_{2}\right)\right]\right\}
\end{aligned}
$$

$$
\begin{gathered}
E_{\mathrm{so}}=\frac{1}{2} W_{0} \int d \boldsymbol{r}\left\{\boldsymbol{J} \cdot \nabla \rho+\boldsymbol{J}_{n} \cdot \nabla \rho_{n}+\boldsymbol{J}_{p} \cdot \nabla \rho_{p}\right\}, \\
E_{\rho}=\int d \boldsymbol{r}\left\{\frac{1}{12} \rho^{\gamma+2} t_{3}\left(1+\frac{x_{3}}{2}\right)\right. \\
\left.-\frac{1}{12} \rho^{\gamma}\left(\rho_{n}^{2}+\rho_{p}^{2}\right) t_{3}\left(\frac{1}{2}+x_{3}\right)\right\} .
\end{gathered}
$$

The stability of the ground-state wave function against a scaling transformation implies $\left.E^{\prime}(\lambda)\right|_{\lambda=1}=0$ (virial theorem) leading to

$$
2 T+3 E_{\delta}+5\left(E_{\mathrm{fin}}+E_{\mathrm{so}}\right)+(3 \gamma+3) E_{\rho}+E_{C}=0 .
$$

According to Eq. (11), the $m_{3}$ moment of the isoscalar monopole RPA strength can be written as

$$
\begin{gathered}
m_{3}=\frac{1}{2}\left(\frac{2 \hbar^{2}}{m}\right)^{2}\left[2 T+6 E_{\delta}+20\left(E_{\mathrm{fin}}+E_{\mathrm{so}}\right)\right. \\
\left.+(3 \gamma+3)(3 \gamma+2) E_{\rho}\right],
\end{gathered}
$$

where the scaled ground-state energy (14) has been employed. Using Eqs. (20) and (9), the average energy of the ISGMR obtained with the scaling approach reads as

$$
E_{M}^{S}=\sqrt{\frac{m_{3}}{m_{1}}} .
$$

\section{B. Constrained calculation of the giant monopole resonance}

Let us consider a nucleus, described by a Hamiltonian $H$, under the action of a weak one-body field $\eta Q$. Assuming $\eta$ to be sufficiently small so that perturbation theory holds, the variation of the expectation value of $Q$ and $H$ is directly related to the $m_{-1}$ moment [12],

$$
m_{-1}=\sum_{n} \frac{|\langle n|Q| 0\rangle|^{2}}{E_{n}}=-\frac{1}{2}\left[\frac{\partial\langle Q\rangle}{\partial \eta}\right]_{\eta=0}=\frac{1}{2}\left[\frac{\partial^{2}\langle H\rangle}{\partial \eta^{2}}\right]_{\eta=0} .
$$

At the RPA level, the average energy of the ISGMR can also be estimated by performing constrained spherical HF calculations, i.e., by looking for the HF solutions of the constrained Hamiltonian

$$
H(\eta)=H-\eta Q,
$$

where $Q=\sum_{i=1}^{A} r_{i}^{2}$ is the collective monopole operator. From the HF ground-state solution $\Phi(\eta)$ of the constrained Hamiltonian (23), the RPA $m_{-1}$ moment (polarizability) is computed as

$$
\begin{aligned}
m_{-1} & =-\frac{1}{2}\left[\frac{\partial}{\partial \eta}\langle\Phi(\eta)|Q| \Phi(\eta)\rangle\right]_{\eta=0} \\
& =\frac{1}{2}\left[\frac{\partial^{2}}{\partial \eta^{2}}\langle\Phi(\eta)|H| \Phi(\eta)\rangle\right]_{\eta=0},
\end{aligned}
$$

from where another estimate of the average energy of the ISGMR is given by

$$
E_{M}^{C}=\sqrt{\frac{m_{1}}{m_{-1}}}
$$


In the following, we will refer to the average energies provided by the scaling method (21) and to the constrained HF calculations (25) as the scaled and constrained energies, respectively. Because the RPA moments fulfill the relations $\sqrt{m_{3} / m_{1}} \geqslant m_{1} / m_{0} \geqslant \sqrt{m_{1} / m_{-1}}[12]$, the values of the average energies $E_{M}^{S}$ and $E_{M}^{C}$ are an upper and lower bound of the mean energy of the resonance, and their difference is related to the variance of the strength function (resonance width)

$$
\sigma=\frac{1}{2} \sqrt{\left(E_{M}^{S}\right)^{2}-\left(E_{M}^{C}\right)^{2}} .
$$

In the RPA formalism, $\sigma$ is the escape width; that includes the Landau damping but not the spreading width coming from more complicated excitation mechanisms. Therefore, the width estimated with (26) can be significantly lower than the experimentally measured value [24].

\section{NUMERICAL RESULTS}

In our study of the excitation energies of the giant monopole resonances in the sum rule approach, we consider the isotopes of some proton magic nuclei, namely, $\mathrm{O}, \mathrm{Ca}, \mathrm{Ni}, \mathrm{Zr}$, and $\mathrm{Pb}$, from the proton up to the neutron drip lines. The calculations are performed using the $\mathrm{SkM}^{*}$ force. The results are presented in the following manner. First we discuss in detail the behavior of the ISGMR along the isotopic $\mathrm{Ca}$ chain for two reasons. First, the shell effects in the $m_{-1}$ sum rule are especially important along this chain. Second, the sum rule estimates for some isotopes $\left({ }^{34} \mathrm{Ca}\right.$ and $\left.{ }^{60} \mathrm{Ca}\right)$ can be contrasted with presently existing full RPA calculations $[17,18]$ in order to learn which kind of information can be derived from the simpler sum rule approach. In light of these discussions of $\mathrm{Ca}$ isotopes, we analyze the ISGMR in the remaining $(\mathrm{O}, \mathrm{Ni}, \mathrm{Zr}$, and $\mathrm{Pb})$ isotopic chains.

\section{A. The sum rule approach in calcium isotopes}

In Fig. 1, we display the scaled $\left(E_{M}^{S}\right)$ and constrained $\left(E_{M}^{C}\right)$ estimates of the average excitation energy of the monopole oscillation as a function of the mass number $A$ from the proton to the neutron drip lines. In this figure, the filled symbols correspond to closed subshell nuclei, whereas the open symbols correspond to open shell nuclei populated according to the uniform filling approximation. The predicted scaled and constrained energies of these open shell nuclei are thus for orientation purposes. Along the Ca chain the scaled monopole energies decrease rather smoothly when the number of neutrons moves from the stable nuclei toward the neutron drip line. The constrained energies also show a similar global tendency. However, two important differences can be observed as compared with the scaled energies. On the one hand, the reduction of $E_{M}^{C}$ near the neutron drip line is more pronounced than that in the case of scaled energies. The constrained energies $E_{M}^{C}$ also decrease for the proton-rich members of the chain while for these nuclei the scaled energies $E_{M}^{S}$ remain close to the corresponding values in stable nuclei. On the other hand, relatively strong changes of the constrained energies can be observed at some subshell closures, in particular for the ones



FIG. 1. (Color online) Excitation energies of the isoscalar giant monopole resonance in the isotopic chain of calcium from scaling (squares) and constrained (circles) calculations as function of the mass number. Estimate of the resonance width (filled triangles) from Eq. (26) is also displayed.

corresponding to neutron numbers $N=52$ and $N=54$, which are reached once the orbits $2 p_{3 / 2}$ and $2 p_{1 / 2}$ are completely filled. The resonance width along the chain, estimated with Eq. (26), is also displayed in Fig. 1. It increases near the neutron and proton drip lines but also shows noticeable oscillations due to the shell effects at $N=32$ and $N=34$ in the middle of the $\mathrm{Ca}$ chain far from the drip lines.

The upper panel of Fig. 2 displays the $m_{3}$ sum rule as a function of the mass number $A$ along the chain of $\mathrm{Ca}$ isotopes. It can be seen that $m_{3}$ smoothly grows with increasing $A$ due to the global character of this sum rule [see Eq. (20)]. More interesting is the sum rule $m_{3}$ divided by $A$, which is shown in the inset of the same panel. The ratio $m_{3} / A$ is roughly constant for the stable nuclei and smoothly decreases when approaching the neutron and proton drip lines. This behavior can be understood in terms of the finite nucleus incompressibility $K_{A}[13]$ which is proportional to $m_{3} / A$. The value of $K_{A}$ can also be estimated through its leptodermous expansion [13]

$$
K_{A}=K_{\infty}+K_{\mathrm{sf}} A^{-1 / 3}+K_{\mathrm{vs}} I^{2}+K_{\mathrm{coul}} Z^{2} A^{-4 / 3}+\cdots,
$$

where $K_{\infty}$ is the nuclear matter incompressibility and $K_{\text {sf }}, K_{\text {vs }}$, and $K_{\text {Coul }}$ are the surface, symmetry, and Coulomb corrections which in the scaling model are negative [13]. Near the neutron drip line, the surface $\left(K_{\mathrm{sf}} A^{-1 / 3}\right)$ and Coulomb $\left(K_{\text {coul }} Z^{2} A^{-4 / 3}\right)$ terms decrease in absolute magnitude and tend to make $K_{A}$ larger with growing $A$, but the contribution of the symmetry term $\left(K_{\mathrm{vs}} I^{2}\right)$ dominates because of the large values of the neutron excess $I=(N-Z) / A$ and, in total, the finite nucleus incompressibility $K_{A}$ is reduced. Near the proton drip line, all three correction terms to $K_{A}$ are larger in absolute magnitude than those for stable nuclei, and thus the finite nucleus incompressibility again decreases. However, this effect near the proton drip line is smaller than that near the neutron drip 




FIG. 2. $m_{3}$ sum rule for calcium isotopes as a function of the mass number $A$ (upper panel). Total $m_{1}$ and $m_{-1}$ sum rules (filled triangles) and contributions coming from neutrons (squares) and protons (circles) (middle and lower panels).

line because of the smaller $|I|$ values, at least in the case of the $\mathrm{Ca}$ isotopes.

In the middle panel of Fig. 2, we display the $m_{1}$ RPA sum rule for the chain of $\mathrm{Ca}$ isotopes as well as their contribution coming separately from protons and neutrons. As can be seen, the proton contribution to $m_{1}$ is roughly constant because the proton rms radius changes only very little along the whole chain. Contrarily, the neutron contribution to $m_{1}$ increases almost linearly with increasing number of neutrons from the proton drip line up to ${ }^{70} \mathrm{Ca}$ from where it starts to grow faster following the trend of the rms radius of the neutron densities near the neutron drip line. Because of this behavior of the proton and neutron contributions, the full $m_{1}$ as a function of $A$ behaves almost as its neutron contribution along the chain. It should be pointed out that near the proton drip line, the proton and neutron contributions are rather similar and both contribute equally to $m_{1}$; while near the neutron drip line, the $m_{1}$ sum rule is basically given by its neutron contribution.

The $m_{-1}$ sum rule for the chain of $\mathrm{Ca}$ isotopes is displayed in the lower panel of Fig. 2 together with their proton and neutron contributions. From this figure, one can see that the neutron contribution to $m_{-1}$ increases almost linearly with mass number $A$ from the proton drip line to ${ }^{48} \mathrm{Ca}$ and from ${ }^{54} \mathrm{Ca}$ to ${ }^{70} \mathrm{Ca}$ with sudden changes from ${ }^{48} \mathrm{Ca}$ to ${ }^{54} \mathrm{Ca}$ and from ${ }^{70} \mathrm{Ca}$ up to the neutron drip line nucleus ${ }^{76} \mathrm{Ca}$ (shown in the inset). In contrast, the proton contribution to $m_{-1}$ remains roughly constant except from ${ }^{40} \mathrm{Ca}$ to the proton drip line $\left({ }^{34} \mathrm{Ca}\right)$, where it slightly increases. This behavior of the $m_{-1}$ sum rule, together with the one exhibited by the $m_{1}$ sum rule discussed previously, explains the global trends shown by the

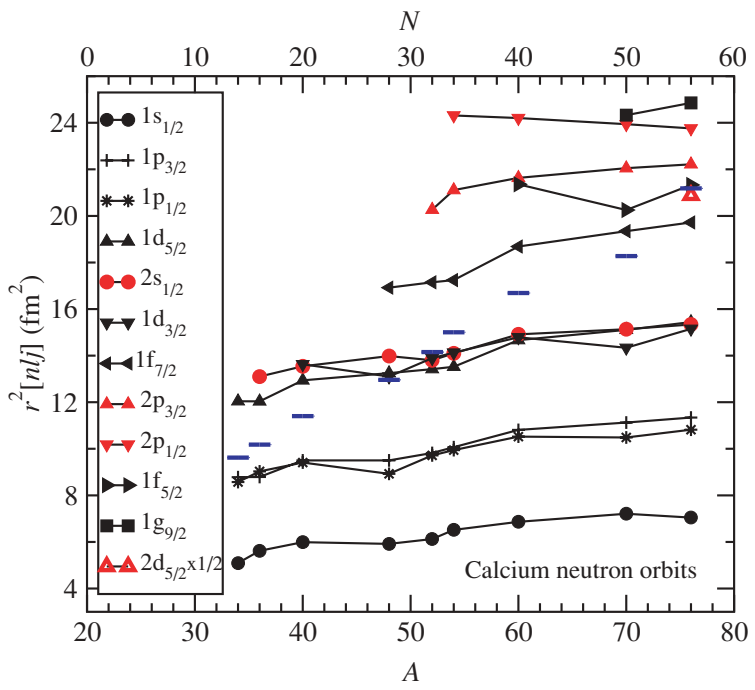

FIG. 3. (Color online) Mean square radii of the neutron singleparticle orbits for the isotopic chain of calcium as a function of the mass number. Horizontal bars depict the value of the total neutron mean square radius of each isotope.

constrained average energy $E_{M}^{C}$ of the ISGMR along the Ca chain displayed in Fig. 1 from the proton to the neutron drip lines.

To obtain more insight about the neutron contribution to the $m_{1}$ and $m_{-1}$ sum rules, we display in Fig. 3 the mean square radius of each neutron orbit $n l j$ entering in the HF wave function of the ground state of the $\mathrm{Ca}$ nuclei along the isotopic chain. They can be contrasted with the neutron mean square radii of the same nuclei, shown by the thick horizontal lines. To help the discussion and for further purposes, the neutron and proton single-particle energy levels of the $\mathrm{Ca}$ isotopes are displayed in Figs. 4 and 6, respectively. In these figures, we also included some quasi-bound levels owing to the centrifugal (neutrons) or centrifugal plus Coulomb (protons) barriers which simulate possible positive-energy single-particle resonant states. From Fig. 3, we can see that the deeper orbits almost give the same mean square radius along the whole isotopic chain in agreement with the fact that the more bound energy levels do not change very much from the proton to the neutron drip line (see Fig. 4). Beyond ${ }^{48} \mathrm{Ca}$, neutrons start to be accommodated in levels whose binding energies are relatively small, and consequently the rms radii of the corresponding orbits increase. It is important to note that, in particular, the $2 p_{3 / 2}$ and $2 p_{1 / 2}$ orbits have a very large mean square radius even though their binding energies are not very small, especially for the heaviest isotopes. The mean square radius of these $p$ orbits is larger than that corresponding to the less bound level $1 f_{5 / 2}$, and the one of the $2 p_{1 / 2}$ orbit is similar to that of the $1 g_{9 / 2}$ orbit which lies higher in the energy spectrum. Near the neutron drip line, the very lightly bound level $2 d_{5 / 2}$ is occupied, and its wave function extends rather farther, increasing substantially the mean square radius of ${ }^{76} \mathrm{Ca}$ (the contribution of the $2 d_{5 / 2}$ level to the mean square radius is shown by a triangle in Fig. 3 in the reduced scale as indicated). This fact produces a noticeable departure from the 




FIG. 4. (Color online) Neutron single-particle energies for calcium isotopes as a function of $A$. Symbols joined by dotted lines correspond to empty levels.

linear behavior exhibited by the $m_{1}$ sum rule near the neutron drip line.

In Fig. 5, we display the contributions $m_{-1}[n l j]$ of a single nucleon in the occupied neutron orbits to the total $m_{-1}$ sum rule for each $\mathrm{Ca}$ isotope. This figure shows that the more bound neutron levels give almost the same single-particle contribution to $m_{-1}$ in all the nuclei of the $\mathrm{Ca}$ chain. The behavior of the $m_{-1}$ sum rule from ${ }^{48} \mathrm{Ca}$ onward to the neutron drip line is governed by the outer orbits $2 p_{3 / 2}, 2 p_{1 / 2}, 1 f_{5 / 2}, 1 g_{9 / 2}$, and $2 d_{5 / 2}$, which give the largest contribution to $m_{-1}$ from ${ }^{52} \mathrm{Ca}$ to ${ }^{76} \mathrm{Ca}$. The single-particle contributions to $m_{-1}$ from the orbits $2 p_{3 / 2}$ and $1 g_{9 / 2}$ are still roughly constant; however, strong changes can be observed in $m_{-1}[n l j]$ for the $2 p_{1 / 2}$ and $1 f_{5 / 2}$ orbits. A very large $m_{-1}[n l j]$ value comes from the outermost very lightly bound orbital $2 d_{5 / 2}$ in the drip line

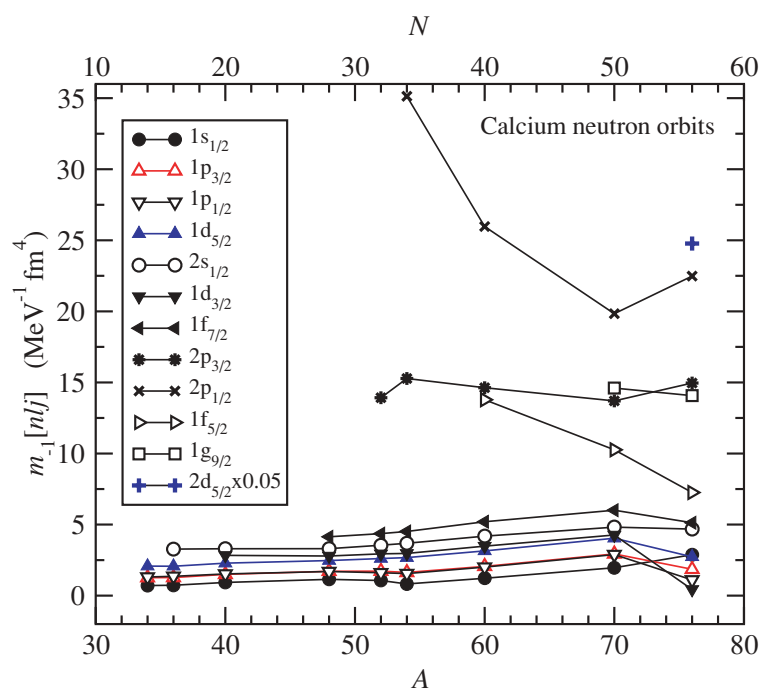

FIG. 5. (Color online) Contributions of a single neutron in each occupied orbit to the $m_{-1}$ sum rule for the calcium isotopes.

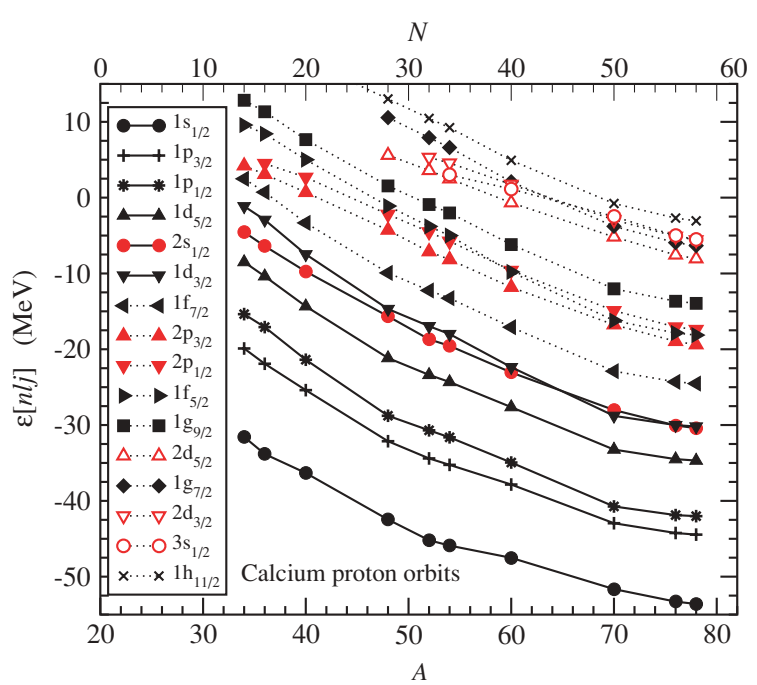

FIG. 6. (Color online) Same as Fig. 4, but for protons.

nucleus ${ }^{76} \mathrm{Ca}$ (shown as a plus sign in the figure in the reduced scale as indicated). The large single-particle contribution of the $2 p_{1 / 2}$ and $2 d_{5 / 2}$ orbits to $m_{-1}$ is due to the extension of their corresponding wave functions beyond the core of the nucleus, so that neutrons in such orbitals are much softer to pick up than neutrons accommodated in inner orbitals producing the strong enhancement of the total $m_{-1}$ sum rule.

Let us now discuss the enhancement of the $m_{-1}$ sum rule near the proton drip line. As it is known [30], the depth of the single-particle potential for protons decreases when the number of neutrons in an isotopic chain decreases. Thus the outermost bound proton levels for nuclei near the proton drip line (see Fig. 6) have little binding energy and their corresponding HF wave functions extend far from the core of the nuclei; consequently, protons in these orbits are softer against pickup thereby increasing the contribution to the total $m_{-1}$ sum rule. This is just the case of the $1 d_{3 / 2}$ orbit for protons in $\mathrm{Ca}$ isotopes (see Fig. 6), which passes from $-7.5 \mathrm{MeV}$ in ${ }^{40} \mathrm{Ca}$ to $-1.1 \mathrm{MeV}$ in the proton drip line nucleus ${ }^{34} \mathrm{Ca}$ and gives the largest contribution to $m_{-1}$ in this case.

\section{B. Connection with the RPA strength function}

As mentioned, the sum rules $m_{1}, m_{3}$, and $m_{-1}$ computed from the HF ground state by means of Eqs. (9), (20), and (24), are the exact $1 p$ - $1 h$ RPA value [12]. Actually, the selfconsistent HF sum rules provide a practical means to check the accuracy of an RPA calculation of the strength function $S(E)$, provided that both the sum rule and the RPA calculation are performed in the same conditions. However, because of their complexity, the RPA calculations often are not fully self-consistent. For instance, the Coulomb and/or spin-orbit residual interactions may be absent in the RPA response. As recently shown in [31], when the RPA calculations are performed self-consistently, the sum rules extracted from the RPA strength function are quite close to the values obtained in the HF sum rule approach. 
RPA calculations for ${ }^{34} \mathrm{Ca}$ and ${ }^{60} \mathrm{Ca}$ were carried out some time ago [17]. The extracted ISGMR mean energies were $E_{M}^{S}=22.5$ and $E_{M}^{C}=17.8 \mathrm{MeV}$ for ${ }^{34} \mathrm{Ca}$, and $E_{M}^{S}=18.8$ and $E_{M}^{C}=15.4 \mathrm{MeV}$ for ${ }^{60} \mathrm{Ca}$. Although there are some differences, they compare well with the values from our sum rule calculation: $E_{M}^{S}=22.0$ and $E_{M}^{C}=17.3 \mathrm{MeV}$ for ${ }^{34} \mathrm{Ca}$, and $E_{M}^{S}=18.3$ and $E_{M}^{C}=15.0 \mathrm{MeV}$ for ${ }^{60} \mathrm{Ca}$. Our values for the resonance widths are $6.8 \mathrm{MeV}$ in ${ }^{34} \mathrm{Ca}$ and $5.3 \mathrm{MeV}$ in ${ }^{60} \mathrm{Ca}$, which coincide with the RPA values obtained in Ref. [17]. The systematic deviation of about $0.5 \mathrm{MeV}$ of the RPA average energies reported in Ref. [17] from our sum rule results may possibly be attributed to a deficiency in full self-consistency.

The information contained in the energy spectra of the nuclei as well as in the values of the sum rules allows one to infer important properties of the strength function $S(E)$. Looking at Fig. 10 of Ref. [17], one can see that for ${ }^{34} \mathrm{Ca}$ there is a low-energy bump in the region around $5-13 \mathrm{MeV}$. It is mainly due to transitions from the less bound proton levels $\left(1 d_{3 / 2}\right.$ and $\left.2 s_{1 / 2}\right)$ to the continuum. These transitions enhance the $m_{-1}$ sum rule that weighs the low energy part of $S(E)$. The peak seen at high energies, in the $19-25 \mathrm{MeV}$ region, receives contributions from the $1 p \rightarrow 2 p$ (resonant) transition of protons as well as from the $1 p \rightarrow 2 p$ and $1 s \rightarrow 2 s$ transitions of neutrons. Concerning ${ }^{60} \mathrm{Ca}$ (Fig. 12 of Ref. [17]), the low-energy bump around $4-12 \mathrm{MeV}$ is again mainly related to transitions to the continuum from the less bound levels, which in this case are due to neutrons. For this nucleus the last neutron in the $1 f_{5 / 2}$ orbit is bound by $3.4 \mathrm{MeV}$ which is in agreement with the threshold energy. The high-energy peaks around $17 \mathrm{MeV}$ and $21 \mathrm{MeV}$ can be related to the $2 s \rightarrow 3 s$ (virtual) and to the $1 d_{5 / 2} \rightarrow 2 d_{5 / 2}$ (resonant) transitions for protons and to the $1 p \rightarrow 2 p, 1 d_{5 / 2} \rightarrow 2 d_{5 / 2}$, and $1 d_{3 / 2} \rightarrow 2 d_{3 / 2}$ (resonant) transitions for neutrons. Strictly speaking, these transitions between single-particle levels, should be related to peaks of the unperturbed strength, but they also appear when the RPA correlations are included. They are, however, slightly shifted to lower energies in general. With this experience in hand, we will discuss from a qualitative point of view the most important trends of the RPA monopole response which can be expected for some representative nuclei of the $\mathrm{Ca}$ isotopic chain.

For ${ }^{52} \mathrm{Ca}$ and ${ }^{54} \mathrm{Ca}$, a low-energy bump in $S(E)$ starts to be developed because of transitions from $2 p_{3 / 2}$ and $2 p_{1 / 2}$ levels to the continuum. Although the strength in the low-energy region of $S(E)$ should be small because of the relatively large binding energy of the last filled levels of neutrons of these nuclei (6.7 and $4.1 \mathrm{MeV}$ for ${ }^{52} \mathrm{Ca}$, and ${ }^{54} \mathrm{Ca}$, respectively), the large rms radii of these orbits favor a large overlap between the wave functions of these bound levels and the levels in the continuum [32], which increases the strength at these energies. This fact is supported, on average, by the enhancement of the $m_{-1}$ sum rule in passing from ${ }^{48} \mathrm{Ca}$ to ${ }^{54} \mathrm{Ca}$. This confirms our assumption about the key role of the $2 p_{3 / 2}$ and $2 p_{1 / 2}$ orbits on the sudden increase of $m_{-1}$. In passing from ${ }^{60} \mathrm{Ca}$ to ${ }^{70} \mathrm{Ca}$, the increase of $m_{1}$ and $m_{-1}$ is basically a size effect which explains the almost similar values of $E_{M}^{C}$ in both nuclei. Here one should also expect a low-energy bump shifted toward lower energies because of transitions to the continuum from the $1 g_{9 / 2}$ orbit, which is bound by $2.5 \mathrm{MeV}$. For ${ }^{76} \mathrm{Ca}, m_{-1}$ strongly increases as a result of the effect of the $2 d_{5 / 2}$ orbit (bound by only $0.84 \mathrm{MeV}$ ) which has a very large rms radius. Although $m_{1}$ slightly increases, the large change of $m_{-1}$ explains the dramatic reduction of $E_{M}^{C}$ at the neutron drip line for the $\mathrm{Ca}$ isotopes. For this nucleus, one could also expect a large low-energy bump dominated by the transition to the continuum from the $2 d_{5 / 2}$ level. The structure in $S(E)$ should also contain broad high-energy peaks due to transitions from the deeply bound states to the unoccupied bound or resonant levels, coming from both neutrons and protons. From this it appears that knowledge of the spectrum of a nucleus as well as the $m_{1}$ and $m_{-1}$ sum rules can provide useful information that allows one to sketch the behavior of the strength distribution $S(E)$.

\section{Transition densities}

In this subsection, we will discuss the two transition densities [15]

$$
\delta \rho_{+1}(\boldsymbol{r})=-\frac{1}{2 A\left\langle r^{2}\right\rangle}\left[3 \rho(\boldsymbol{r})+r \rho^{\prime}(\boldsymbol{r})\right],
$$

which is the Tassie transition density corresponding to the scaling transformation, and

$$
\delta \rho_{-1}(\boldsymbol{r})=-\left.\frac{1}{2 m_{-1}} \frac{\partial \rho_{\eta}(\boldsymbol{r})}{\partial \eta}\right|_{\eta=0}
$$

which is the transition density in the constrained case. In Eq. (28), $\rho(\boldsymbol{r})$ is the ground-state particle density and $\left\langle r^{2}\right\rangle$ the mean square radius of the nucleus. In Eq. (29), $\rho_{\eta}(\boldsymbol{r})$ is the particle density built up with the HF single-particle wave functions that are solutions of the constrained Hamiltonian (23), and $m_{-1}$ is the inverse energy-weighted sum rule calculated using (24). The chosen normalization in Eqs. (28) and (29) ensures that when a single state exhausts the sum rule, then $\delta \rho_{+1}(\boldsymbol{r})=\delta \rho_{-1}(\boldsymbol{r})$.

Figure 7 displays the neutron (upper panel) and proton (middle panel) contributions to the constrained transition densities (29) multiplied by $r^{2}$ for some Ca isotopes between the proton and neutron drip lines, namely ${ }^{34} \mathrm{Ca},{ }^{48} \mathrm{Ca},{ }^{54} \mathrm{Ca}$, ${ }^{70} \mathrm{Ca}$, and ${ }^{76} \mathrm{Ca}$. The neutron contribution to $r^{2} \delta \rho_{-1}$ strongly depends on the neutron number of the considered isotope. The outer bump of the neutron contribution broadens and shifts to larger values of $r$ when the nuclei approach the neutron drip line; this is basically due to the neutrons in the outermost orbits which are loosely bound and extend very far from the center of the nucleus. For example, in Fig. 7 one can see the effect of the $2 p_{3 / 2}$ and $2 p_{1 / 2}$ orbits in ${ }^{54} \mathrm{Ca}$ where the outer bump is clearly shifted with respect to that of the ${ }^{48} \mathrm{Ca}$ nucleus. The fact that the rms radius of the $1 f_{5 / 2}$ and $1 g_{9 / 2}$ orbits are similar to those of the $2 p_{3 / 2}$ and $2 p_{1 / 2}$ orbits (see Fig. 3 ) is also reflected in the transition density of the nucleus ${ }^{70} \mathrm{Ca}$, whose external bump is located, roughly, at the same position as that of the one corresponding to ${ }^{54} \mathrm{Ca}$. The very lightly bound $2 d_{5 / 2}$ orbit has a very large rms radius (see Fig. 3), and consequently the external bump of the neutron contribution to the constrained transition density is pushed to very large distances. The proton contribution to the constrained transition density displayed in the middle panel of Fig. 7 


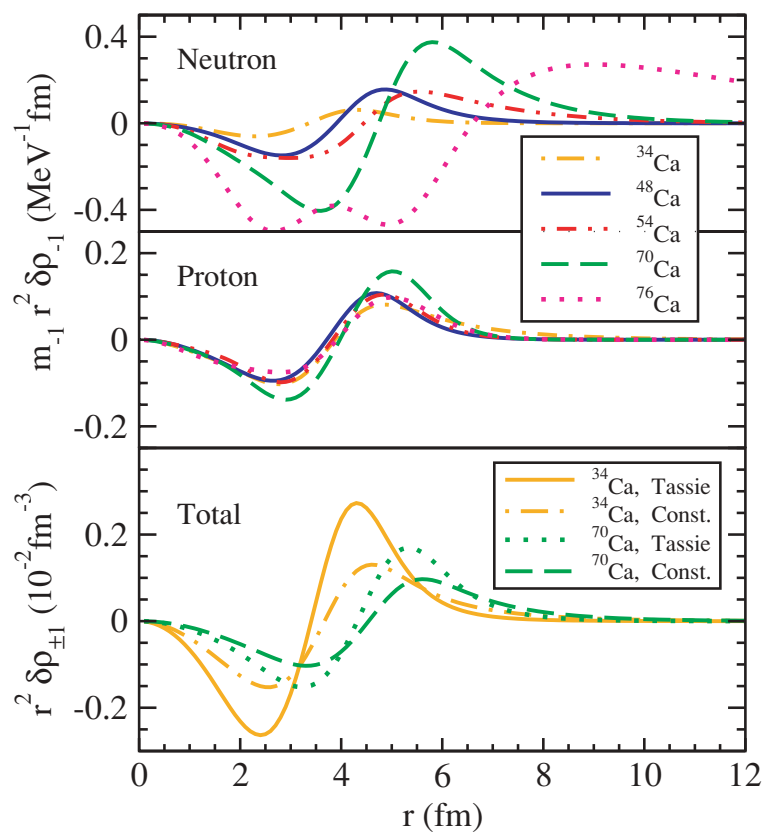

FIG. 7. (Color online) Spatial variation of the constrained transition densities for neutrons (upper panel) and protons (middle panel) of some selected $\mathrm{Ca}$ isotopes. The Tassie and constrained transition densities are compared in the lower panel. Notice the different vertical scales.

shows a completely different pattern. On the one hand, both the inner and outer bumps are located approximately at the same positions for all the considered $\mathrm{Ca}$ isotopes, which is in agreement with the fact that the proton rms radius along a given isotopic chain remains approximately constant. On the other hand, the proton contribution to the total transition density decreases with increasing mass number. This is consistent with the fact that protons contribute proportionally less than neutrons to the $m_{-1}$ sum rule in neutron drip line nuclei as can be seen from the lower panel of Fig. 2.

The lower panel of Fig. 7 compares the Tassie and the constrained transition densities for the drip line nuclei, ${ }^{34} \mathrm{Ca}$ and ${ }^{70} \mathrm{Ca}$. For both nuclei, the bumps of $r^{2} \delta \rho_{+1}$ and $r^{2} \delta \rho_{-1}$ are located at the same place. However, the shapes of both transition densities differ relatively between them. The strong fragmentation of the RPA strength in $\mathrm{Ca}$ isotopes, in particular in the drip line nuclei, can be gauged by the large enhancement of the resonance width in these nuclei (see Fig. 1).

\section{Other isotopic chains}

With the experience gained from this analysis of $\mathrm{Ca}$ isotopes, let us discuss the more salient features found in other isotopic chains. Figure 8 displays the scaled $E_{M}^{S}$ and constrained $E_{M}^{C}$ energies of the ISGMR as well as the resonance width for the $\mathrm{O}, \mathrm{Ni}, \mathrm{Zr}$, and $\mathrm{Pb}$ isotopic chains. The filled and open symbols correspond to subshell closed and open nuclei, respectively. The scaled energies along these isotopic chains qualitatively behave in a similar way as those in $\mathrm{Ca}$ isotopes. In general, $E_{M}^{S}$ is roughly constant along the considered chains from the proton drip line to the stable nuclei



FIG. 8. (Color online) Excitation energy of ISGMR from scaling and constrained calculations, and predicted resonance width for the isotopic chains of $\mathrm{O}, \mathrm{Ni}, \mathrm{Zr}$, and $\mathrm{Pb}$.

and then shows a moderate downward trend while approaching the neutron drip line. On the contrary, the constrained energies exhibit again a sizable reduction near the neutron drip line and a moderate decrease near the proton drip line. One exception to this general behavior is ${ }^{12} \mathrm{O}$, which shows a noticeable decrease of the scaled and constrained energies at the proton drip line. The reasons are similar to those discussed for ${ }^{34} \mathrm{Ca}$, but more accentuated because of the smaller nuclear charge. Another exception is the heavy $\mathrm{Pb}$ chain where the constrained energy $E_{M}^{C}$ smoothly diminishes from the proton to the neutron drip lines showing a similar behavior to that of the scaled energy for these isotopes. As a consequence, for light and medium isotopic chains $(\mathrm{O}, \mathrm{Ni}$, and $\mathrm{Zr})$ the ISGMR width shows an enhancement near the proton and neutron drip lines (somewhat more pronounced at the neutron drip line), whereas the resonance widths for the $\mathrm{Pb}$ isotopes remain approximately constant along the whole chain. Thus, the resonance width in the different isotopic chains shows a downward tendency with increasing atomic number, which suggests a smaller fragmentation and more collective character in the ISGMR of heavy nuclei such as the $\mathrm{Pb}$ isotopes.

We now discuss the $m_{1}$ and $m_{-1}$ sum rules in $\mathrm{O}, \mathrm{Ni}$, $\mathrm{Zr}$, and $\mathrm{Pb}$ isotopes. In Figs. 9 and 10 we display $m_{1} / A^{5 / 3}$ and $m_{-1} / A^{7 / 3}$, respectively, for the aforementioned isotopic chains. These scaling factors $A^{-5 / 3}$ and $A^{-7 / 3}$ remove the size dependence of the total $m_{1}$ and $m_{-1}$ sum rules [24]. Figure 9 shows that the neutron contribution to $m_{1} / A^{5 / 3}$ increases and the proton contribution decreases with growing mass number $A$. However, the total $m_{1} / A^{5 / 3}$ displays a rather constant behavior as a function of $A$ for all the considered isotopic chains, except maybe for some drip line nuclei. This means that the enhancement of the $m_{1}$ sum rule with $A$ displayed in Fig. 2 for $\mathrm{Ca}$ isotopes up to ${ }^{70} \mathrm{Ca}$ is basically due to a size effect except near the drip line nuclei. The $A$-scaled $m_{-1} / A^{7 / 3}$ sum rule displayed in Fig. 10 for the $\mathrm{O}, \mathrm{Ni}, \mathrm{Zr}$, and $\mathrm{Pb}$ chains shows a rather constant behavior in the region of stable nuclei. It clearly increases near the neutron drip lines and also for the proton drip line of oxygen. It shows the softness against 


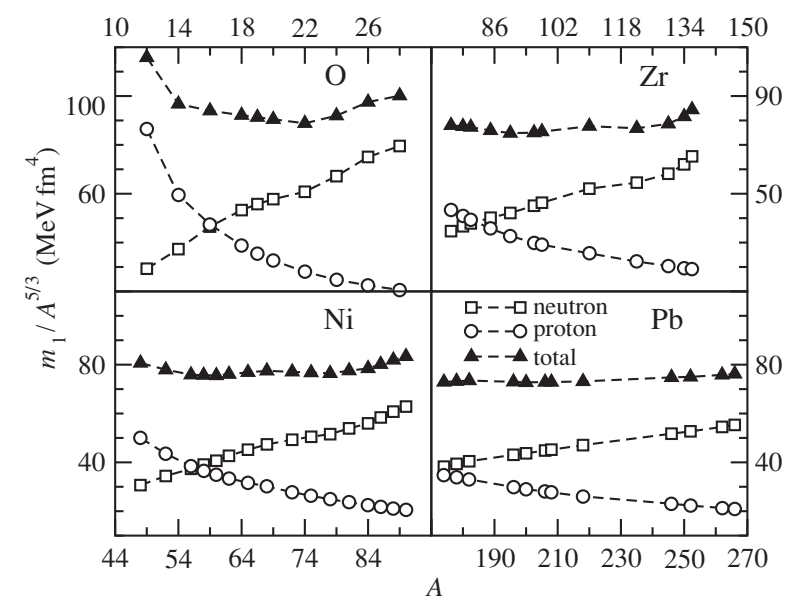

FIG. 9. $m_{1}$ sum rule scaled by $A^{-5 / 3}$ for the isotopic chains of $\mathrm{O}$, $\mathrm{Ni}, \mathrm{Zr}$, and $\mathrm{Pb}$. Neutron (squares) and proton (circles) contributions to the total $m_{1}$ are also plotted.

pick-up of the nucleons from the outermost lightly bound levels thus giving the large enhancement of the $m_{-1}$ sum rule in the case of drip line nuclei.

From the sum rule approach, we can also obtain some information about the RPA strength distributions for $\mathrm{O}, \mathrm{Ni}$, and $\mathrm{Pb}$ isotopes. Reference [33] studied the isoscalar monopole RPA strength for the neutron drip line nucleus ${ }^{28} \mathrm{O}$. We can analyze this nucleus on the basis of the sum rule approach. To help the discussion, we report in Table I the neutron and proton single-particle energy levels (including the quasibound ones) as well as the mean square radius of each bound orbit for this nucleus obtained with the $\mathrm{SkM}^{*}$ force. In this case, the last occupied neutron level $\left(1 d_{3 / 2}\right)$ is bound by only 1.8 MeV. As discussed in Ref. [33], the RPA strength develops a low-energy bump because of transitions to the continuum from the $1 d_{5 / 2}, 2 s_{1 / 2}$, and $1 d_{3 / 2}$ levels, and a high-energy peak because of the neutron transition $1 p_{3 / 2} \rightarrow 2 p_{3 / 2}$ (resonant) and the proton transitions from the $1 s_{1 / 2}, 1 p_{1 / 2}$, and $1 p_{3 / 2}$ levels to the unoccupied (but bound) $2 s_{1 / 2}, 2 p_{1 / 2}$, and $2 p_{3 / 2}$ levels, respectively. This structure is actually encoded in the sum rule approach used here. Looking at Fig. 10, a noticeable

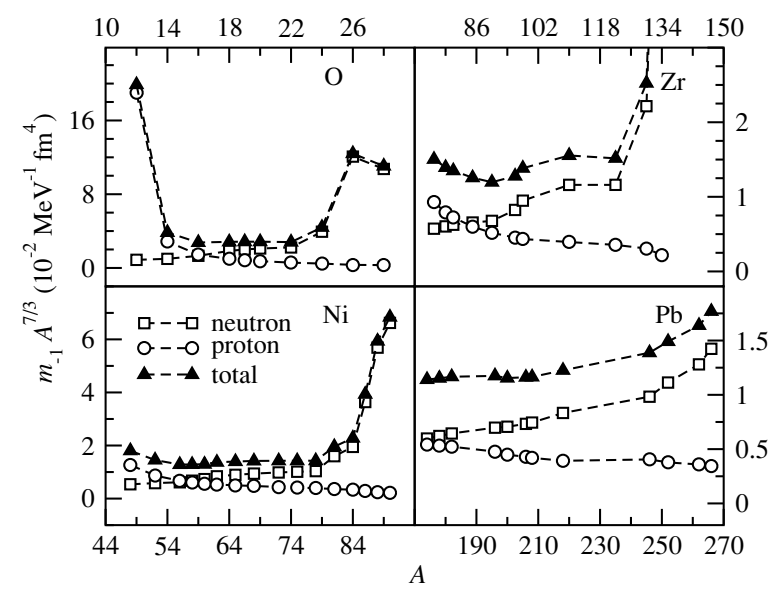

FIG. 10. $m_{-1}$ sum rule scaled by $A^{-7 / 3}$ for the isotopic chains of $\mathrm{O}, \mathrm{Ni}, \mathrm{Zr}$, and $\mathrm{Pb}$. Neutron (squares) and proton (circles) contributions to the total $m_{-1}$ are also plotted.

enhancement of the scaled $m_{-1} / A^{7 / 3}$ sum rule for ${ }^{28} \mathrm{O}$ can be seen in qualitative agreement with the large amount of strength in the low-energy part of $S(E)$. To be more quantitative, we also report in Table I the contribution to $m_{-1}$ coming from a single neutron in each occupied orbit. The largest part of the $m_{-1}$ sum rule comes from contributions of the $1 d_{3 / 2}, 2 s_{1 / 2}$, and $1 d_{5 / 2}$ neutron levels, which are responsible for 74,11 , and $8 \%$ of the total $m_{-1}$ sum rule $\left(263.022 \mathrm{MeV}^{-1} \mathrm{fm}^{4}\right)$, respectively. On the other hand, using the $\left\langle r^{2}\right\rangle$ values reported in the same table, we find that the contribution of these neutron levels to the total $m_{1}$ sum rule $\left(2.586 \times 10^{4} \mathrm{MeV} \mathrm{fm}^{4}\right)$ is 23,11 and $25 \%$, pointing out again their relevance in the low-energy part of $S(E)$.

The single-nucleon contributions to $\left\langle r^{2}\right\rangle$ and to the $m_{-1}$ sum rule reported in Table I can also provide information about $S(E)$. The energy corresponding to the maximum of the unperturbed strength due to transitions of the outermost nucleons to the continuum can be estimated for each orbit as the square root of the ratio of their single-nucleon contributions to $m_{1}[n l j]$ and $m_{-1}[n l j]$. From the values reported in Table I, this estimate gives 5.6 and $9.6 \mathrm{MeV}$ for the $1 d_{3 / 2}$ and

TABLE I. Neutron and proton single-particle energy levels (in $\mathrm{MeV}$ ) with the occupancy (given in parentheses) for ${ }^{28} \mathrm{O}$. The mean square radius $\left\langle r^{2}\right\rangle$ (in $\mathrm{fm}^{2}$ ) and the single-nucleon contribution to the $m_{-1}$ sum rule of each orbital (in $\mathrm{MeV}^{-1}$ $\mathrm{fm}^{4}$ ) are also given.

\begin{tabular}{|c|c|c|c|c|c|c|c|}
\hline \multicolumn{4}{|c|}{ Neutron } & \multicolumn{4}{|c|}{ Proton } \\
\hline Orbital & Energy & $\left\langle r^{2}\right\rangle$ & $\overline{m_{-1}[n l j]}$ & Orbital & Energy & $\left\langle r^{2}\right\rangle$ & $m_{-1}[n l j]$ \\
\hline $1 s_{1 / 2}$ & $-31.920(1)$ & 5.085 & 0.3093 & $1 s_{1 / 2}$ & $-44.132(1)$ & 5.433 & 0.3505 \\
\hline $1 p_{3 / 2}$ & $-19.920(1)$ & 8.701 & 0.9664 & $1 p_{3 / 2}$ & $-31.726(1)$ & 8.786 & 1.0010 \\
\hline $1 p_{1 / 2}$ & $-14.936(1)$ & 9.194 & 1.5875 & $1 p_{1 / 2}$ & $-27.060(1)$ & 9.068 & 1.3510 \\
\hline $1 d_{5 / 2}$ & $-8.641(1)$ & 12.962 & 3.6757 & $1 d_{5 / 2}$ & $-19.389(0)$ & - & - \\
\hline $2 s_{1 / 2}$ & $-5.997(1)$ & 16.689 & 15.1465 & $2 s_{1 / 2}$ & $-15.143(0)$ & - & - \\
\hline $1 d_{3 / 2}$ & $-1.780(1)$ & 18.278 & 48.9019 & $1 d_{3 / 2}$ & $-12.415(0)$ & - & - \\
\hline $2 p_{3 / 2}$ & $0.526(0)$ & - & - & $1 f_{7 / 2}$ & $-6.665(0)$ & - & - \\
\hline $1 f_{7 / 2}$ & $1.731(0)$ & - & - & $2 p_{3 / 2}$ & $-2.380(0)$ & - & - \\
\hline $1 g_{9 / 2}$ & $10.366(0)$ & - & - & $2 p_{1 / 2}$ & $-0.701(0)$ & - & - \\
\hline
\end{tabular}


$2 s_{1 / 2}$ neutron levels, in qualitative agreement with the position of the maxima in the unperturbed strength displayed in Fig. 1 of Ref. [33].

For the Ni isotopes, the situation is qualitatively similar to that found for the $\mathrm{Ca}$ isotopic chain, although there are some differences. First, the enhancement of the polarizability shown by $\mathrm{Ca}$ isotopes when $2 p_{3 / 2}$ and $2 p_{1 / 2}$ orbits are occupied ${ }^{52} \mathrm{Ca}$ and ${ }^{54} \mathrm{Ca}$, respectively) is appreciably attenuated in the corresponding $\mathrm{Ni}$ isotopes $\left({ }^{60} \mathrm{Ni}\right.$ and $\left.{ }^{62} \mathrm{Ni}\right)$. This is because for $\mathrm{Ni}$ isotopes, the $1 f_{7 / 2}$ orbit for protons is filled and thus neutrons occupying $2 p_{3 / 2}$ and $2 p_{1 / 2}$ levels are more bound and have smaller rms radii than the corresponding $\mathrm{Ca}$ isotopes. They thus contribute less to the $m_{-1}$ sum rule, as can be realized from Fig. 10, where no significant enhancement of the scaled $m_{-1} / A^{7 / 3}$ sum rule is observed. Second, in $\mathrm{Ca}$ isotopes the strong enhancement of the $m_{-1}$ sum rule and a moderate rise in $m_{1}$ start to be appreciable once the $1 g_{9 / 2}$ orbit has been occupied (which corresponds to ${ }^{70} \mathrm{Ca}$ ) and the very lightly bound $2 d_{5 / 2}$ level starts to be populated (see Fig. 2). Something similar happens for $\mathrm{Ni}$ isotopes beyond ${ }^{78} \mathrm{Ni}$, where the $2 d_{5 / 2}, 3 s_{1 / 2}$, and $2 d_{3 / 2}$ orbits become slightly bound and contribute largely to the enhancement of the $m_{-1}$ (strong) and $m_{1}$ (moderate) sum rules, decreasing of the constrained energy and enhancing of the width of the ISGMR in nuclei between ${ }^{78} \mathrm{Ni}$ and the drip line nucleus ${ }^{90} \mathrm{Ni}$. However, the single-nucleon contribution to the $m_{1}$ and $m_{-1}$ sum rules critically depends on its binding energy and increases very fast when the binding energy approaches zero. In our uniform filling approach and using the $\mathrm{SkM}^{*}$ force, the last occupied level in the drip nucleus ${ }^{76} \mathrm{Ca}\left(2 d_{5 / 2}\right)$ is bound by only $0.85 \mathrm{MeV}$, while in the $\mathrm{Ni}$ drip nucleus ${ }^{90} \mathrm{Ni}$ the outermost levels $2 d_{5 / 2}, 3 s_{1 / 2}$, and $2 d_{3 / 2}$ are bound by $3.45,2.26$, and $1.09 \mathrm{MeV}$, respectively. These differences in the binding energies of the last occupied levels explain why the decrease in the constrained energy and the enhancement of the width of the ISGMR is more important in the Ca isotopes than in the $\mathrm{Ni}$ isotopes near the neutron drip line.

We next discuss the chain of the $\mathrm{Zr}$ isotopes. For the ${ }^{122} \mathrm{Zr}$ nucleus, where the neutron shell $N=82$ is closed, the binding energy of the last bound orbit $\left(1 h_{9 / 2}\right)$ is $5.01 \mathrm{MeV}$ with the $\mathrm{SkM}^{*}$ force. Some neutron orbits belonging to the $N=126$ closed shell, namely, the $2 f_{7 / 2}, 3 p_{3 / 2}$, and $3 p_{1 / 2}$ orbitals, become very lightly bound in the ${ }^{130} \mathrm{Zr},{ }^{134} \mathrm{Zr}$, and ${ }^{136} \mathrm{Zr}$ isotopes and thus increase greatly the $m_{1}$ and $m_{-1}$ sum rules in these nuclei close to the neutron drip line. In the uniform filling approximation, such a scenario can be appreciated in Figs. 9 and 10 from the departure from the roughly constant value of the $A$-scaled $m_{1} / A^{5 / 3}$ and $m_{-1} / A^{7 / 3}$ sum rules in subshell closed $\mathrm{Zr}$ isotopes beyond ${ }^{122} \mathrm{Zr}$. The strong enhancement of the $m_{-1}$ sum rule combined with the moderate rise in the $m_{1}$ sum rule produces the dramatic decrease of the constrained energy $E_{M}^{C}$ and the large increase of the resonance width near the neutron drip line, which can be observed in Fig. 8.

The situation is different in the heavy $\mathrm{Pb}$ isotopic chain. In this case, the neutron drip line is reached when the $N=$ 184 shell is completely occupied (the last filled orbit $3 d_{3 / 2}$ is bound by $2.58 \mathrm{MeV}$ ). In the lighter $\mathrm{Ca}, \mathrm{Ni}$, and $\mathrm{Zr}$ isotopic chains, the shell gap above the $N=50$ and $N=82$ closed shells is strongly reduced when approaching the neutron drip line (and even disappears in the case of the $\mathrm{Ca}$ isotopes [6]) as compared to those of the stable nuclei of these chains. Therefore, in nuclei near the neutron drip line, some levels of the next major shell can still be bound before reaching it. However, in neutron-rich $\mathrm{Pb}$ isotopes, the shell closure at $N=184$ is more robust, and no bound levels belong to the next major shell in the neutron drip nucleus. The $A$-scaled $m_{1} / A^{5 / 3}$ sum rule is practically constant along the whole chain and $m_{-1} / A^{7 / 3}$ shows a moderate increasing tendency near the neutron drip line (see Figs. 9 and 10). The combination of these two effects causes the constrained energy and the energy width of the ISGMR in neutron-rich $\mathrm{Pb}$ isotopes to vary only slightly as compared to the corresponding values in the stable nuclei of the chain, as seen in Fig. 8.

We have treated all nuclei in the spherical approximation. This is justified to a large extent because of the semimagic character of the isotopic chains considered. However, some of the isotopes may happen to be deformed, specially in the $\mathrm{Zr}$ chain. For more than two decades, there has been experimental evidence [34,35] that the isoscalar monopole strength in deformed nuclei shows an additional lower energy peak when compared to that of spherical nuclei. This is interpreted as an effect of the coupling between the ISGMR and the isoscalar giant quadrupole resonance in deformed nuclei that broadens and splits the ISGMR strength into two components [36,37]. We believe that this effect may be enhanced in deformed isotopes away from the valley of stability, because the broader distribution of the resonance strengths near the drip lines possibly contributes to an increase in the coupling between the two excitation modes.

\section{SEMICLASSICAL EXTENDED THOMAS-FERMI CALCULATIONS}

As mentioned in the Introduction, we know that for stable nuclei, semiclassical approximations of Thomas-Fermi (TF) type and its extensions (ETF) used with the sum rule approach provide a good estimate of the ISGMR excitation energies at least for stable nuclei [20,24-27]. In this section, we want to study whether these semiclassical approximations are also able to follow the average trend of the quantal results in regions away from the stability line.

To describe semiclassically (at TF or ETF levels) the ISGMR excitation energies with the scaling method, we will calculate first the ground-state neutron and proton densities. This is done by replacing the quantal kinetic energy density $\tau=\sum_{i=1}^{A}\left|\nabla \phi_{i}\right|^{2}$ by the semiclassical one at the TF level $\tau_{\mathrm{TF}}=\sum_{q=n, p} 3 / 5\left(3 \pi^{2}\right)^{2 / 3} \rho_{q}^{5 / 3}$ or at the ETF level including $\hbar^{2}$ or $\hbar^{4}$ corrections [20,25] into the Skyrme energy density functional and then solving the variational Euler-Lagrange equations for the neutron and proton densities $\rho_{n}$ and $\rho_{p}$, respectively [25]. The ETF method introduces $\hbar^{2}\left(\hbar^{4}\right)$ corrections on top of the simple TF kinetic energy through an expansion of second (fourth) order gradients of the particle density, which include nonlocal contributions coming from the spin-orbit potential and the effective mass. These $\hbar$ corrections modify the asymptotic behavior of the self-consistent solution 
of the energy density and give a semiclassical density profile which averages the shell oscillations of the quantal HF density in the bulk and reproduces its falloff at the surface. When $\hbar^{4}$ corrections are taken into account, the semiclassical energy of the ground state becomes roughly similar to the one obtained using the more cumbersome Strutinsky average method [25].

In the semiclassical approaches, the neutron (proton) drip line is reached when the neutron (proton) chemical potential vanishes. We have checked [38] that the neutron drip lines for $\mathrm{Ca}$ and $\mathrm{Pb}$ at $\mathrm{TF}$ level using the $\mathrm{SkM}^{*}$ force correspond to neutron numbers of 48 and 195 which are in reasonable agreement with the HF predictions (56 and 184, respectively). This fact points out that nuclei near the drip lines can be studied through semiclassical methods of the ETF type. If the TF or ETF densities are used to compute (19), the virial theorem is, of course, strictly fulfilled thanks to the self-consistency. The semiclassical sum rules $m_{3}$ and $m_{1}$, which are needed to obtain the excitation energy of the ISGMR in the scaling method, are calculated using again Eqs. (20) and (9) but with the semiclassical self-consistent neutron and proton densities instead of the HF ones.

As it happens in the quantal case, the excitation energy of the ISGMR can also be estimated semiclassically by performing TF or ETF constrained calculations. In this case, one has to minimize the constrained semiclassical energy

$$
\int d \boldsymbol{r}\left[\mathcal{H}_{\mathrm{TF}, \mathrm{ETF}}-\eta r^{2} \rho_{\eta}\right]=E(\eta)-\eta \int d \boldsymbol{r} r^{2} \rho_{\eta},
$$

where the quantal kinetic energy has been replaced by the TF or ETF equivalents. To obtain the semiclassical $m_{-1}$ sum rule, we use Eq. (22) but replace the HF expectation values by the semiclassical ones.

Thus, the semiclassical average excitation energies of the ISGMR are obtained from the RPA sum rules but with the HF expectation values entering in them replaced by the TF or ETF ones. The semiclassical excitation energies of the ISGMR calculated with the Skyrme $\mathrm{SkM}^{*}$ force using the scaling method and performing semiclassical constrained calculations are displayed in the upper panel of Fig. 11 in comparison with the exact RPA average energies $\bar{E}_{3}=\sqrt{m_{3} / m_{1}}$ and $\bar{E}_{1}=$ $\sqrt{m_{1} / m_{-1}}$ (which are the quantal scaled $E_{M}^{S}$ and constrained $E_{M}^{C}$ energies described in Sec. 2) along the $\mathrm{Ca}$ isotopic chain from the proton to the neutron drip lines. The semiclassical predictions of the excitation energies displayed in Fig. 11 are calculated at the pure TF level (dashed-dotted lines) and the ETF level including $\hbar^{2}$ (solid lines) and $\hbar^{4}$ (dashed lines) corrections. From this figure, we can see that the general trends of the excitation energies $\bar{E}_{3}$ of the ISGMR are well reproduced by all the considered semiclassical approximations along the whole isotopic chain. The agreement between the RPA $\bar{E}_{3}$ energies and their semiclassical counterpart improves by increasing the order of the $\hbar$ corrections, as can be seen in the upper panel of Fig. 11. If $\hbar^{4}$ corrections are included in the ETF calculation, then the RPA $\bar{E}_{3}$ energies are almost perfectly reproduced.

As far as the scaling approach is concerned, a first conclusion of this analysis is that the semiclassical approach to the RPA $\bar{E}_{3}$ (scaling) energies of the ISGMR agrees very well with the corresponding quantal RPA values not only for

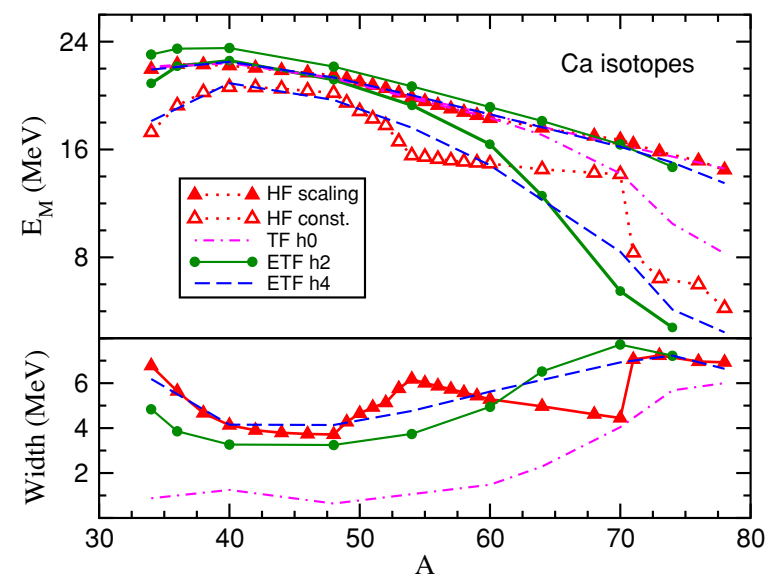

FIG. 11. (Color online) Variation of excitation energies of isoscalar giant monopole resonance with mass number $A$ for $\mathrm{Ca}$ isotopic chain (upper panel). Semiclassical results of Thomas-Fermi (TF) type including $\hbar$ corrections at different orders, obtained with the scaling and constrained approaches, are contrasted with the RPA average energies. Resonance widths (lower panel).

stable nuclei but also for nuclei near the drip lines, specially if $\hbar^{4}$ corrections are included in the semiclassical calculation. This fact points out that the role of shell effects is almost negligible in the estimate of the average energy $\bar{E}_{3}$ of the ISGMR obtained from the $m_{3}$ and $m_{1}$ sum rules on the one hand, and it justifies the use of the liquid-drop-like expansion of the finite nucleus incompressibility (27) along the full isotopic chain on the other hand.

However, the situation is different when the ISGMR excitation energy is estimated by performing constrained calculations. In this case, the constrained TF calculations give excitation energies that lie very close to the values obtained with the scaling method for $\mathrm{Ca}$ isotopes and thus fail in reproducing the RPA $\bar{E}_{1}$ energies in nuclei far from stability. Consequently, in the simple TF approximation, the predicted resonance width is very small and cannot reproduce the behavior of the RPA estimates of the width (26) along the chain as it can be seen in the lower panel of Fig. 11, where the RPA widths and their semiclassical equivalents are displayed. The agreement between the RPA average energies $\bar{E}_{1}$ and their semiclassical estimates considerably improves when $\hbar$ corrections are considered explicitly in the calculation of the semiclassical densities. The ETF- $\hbar^{2}$ average energy of the ISGMR obtained by semiclassical constrained calculations qualitatively describes the behavior of the RPA results. A more quantitative agreement, at least from the proton drip to stable nuclei, is found when the average excitation energies are obtained through ETF- $\hbar^{4}$ calculations. Of course, the effects induced by some specific orbitals in the RPA $\bar{E}_{1}$ energies in $\mathrm{Ca}$ isotopes cannot be recovered by the semiclassical approaches. However, they nicely average the RPA results, the average being better when the $\hbar^{4}$ corrections are taken into account in the calculation. A similar situation is found in the estimates of the width of the ISGMR resonance along the isotopic chain, where the semiclassical approaches average again the RPA values, as can be seen in the lower panel of Fig. 11. 


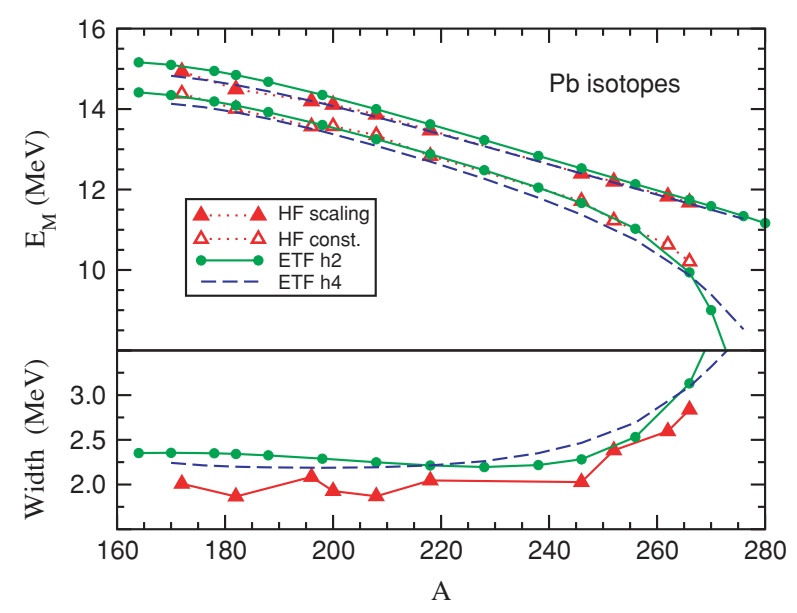

FIG. 12. (Color online) Same as Fig. 11, but for Pb isotopes.

A comparison between the RPA average excitation energies $\bar{E}_{3}$ and $\bar{E}_{1}$ and their semiclassical estimates is displayed in Fig. 12 for the $\mathrm{Pb}$ isotopic chain. As in the case for $\mathrm{Ca}$ isotopes, the semiclassical scaled energies vary smoothly with the mass number $A$ and reproduce quite well the RPA $\bar{E}_{3}$ average energies from the proton to the neutron drip lines, at least for the subshell closed isotopes displayed in the figure. For $\mathrm{Pb}$ isotopes, the RPA $\bar{E}_{1}$ average energies show a rather smooth decreasing tendency with increasing mass number $A$, which is enhanced near the neutron drip line. As happens for $\mathrm{Ca}$ isotopes, the semiclassical $\mathrm{TF}$ constrained energies lie close to the scaled ones and are unable to describe the strong decreasing of $\bar{E}_{1}$ near the neutron drip line. However, when $\hbar$ corrections are added, the semiclassical ETF estimates nicely reproduce the RPA $\bar{E}_{1}$ average energies, specially if $\hbar^{4}$ corrections are taken into account. As discussed before, the ISGMR of $\mathrm{Pb}$ isotopes exhibit a much more collective behavior, and single-particle effects are much less important than in $\mathrm{Ca}$ isotopes even near the drip lines. Thus, it is not completely surprising that the semiclassical approximations of ETF type are able to reproduce accurately not only the $\bar{E}_{3}$ average energies but also the $\bar{E}_{1}$ ones.

\section{SUMMARY AND CONCLUSIONS}

In this paper, we have analyzed the variation of average properties (mean energies and resonance widths) of the isoscalar giant monopole resonance along the isotopic chains between the proton and neutron drip lines for nuclei with magic atomic number from $\mathrm{O}$ to $\mathrm{Pb}$. These average energies are obtained within the RPA sum rule approach using the SkM* interaction. For each nucleus, we have calculated the RPA cubic $\left(m_{3}\right)$ and inverse energy $\left(m_{-1}\right)$ weighted sum rules. The $m_{3}$ sum rule is obtained by means of a scaling transformation of the self-consistent HF neutron and proton densities, while $m_{-1}$ is computed by performing constrained Hartree-Fock calculations. For a Skyrme force, the energy-weighted sum rule $m_{1}$, which is required for evaluating the average energies, is simply proportional to the mean square radius of the Hartree-Fock particle density.

The scaled energies $\bar{E}_{3}$ along the isotopic chains show general trends which are rather independent of the atomic number. The scaled estimate of the average excitation energy of the ISGMR shows a downward tendency as one moves toward the neutron drip line from the stable nuclei. This falloff of the scaled energy is smooth because of the moderate growing behavior of the $m_{3}$ sum rule with increasing mass number which is compensated by the stronger enhancement of the $m_{1}$ sum rule. The latter weighs more the tail of the density distributions and consequently is more important in nuclei near the neutron drip line. This reduction of the scaled estimate of the ISGMR average excitation energy at the neutron drip line is more noticeable in small and medium mass nuclei, and its relative importance decreases with increasing atomic number.

A similar decreasing tendency of $\bar{E}_{3}$ near the proton drip line appears only for very light nuclei such as the $\mathrm{O}$ isotopes, and it is not observed in heavier systems. The reason lies in the fact that for proton drip line nuclei, the Coulomb barrier prevents their single-particle wave functions from extending much from the center of the nucleus, thus prohibiting an enhancement of the rms radius of the density, which in turn would reduce the scaled average excitation energies of these nuclei. Because of the global character of the scaled estimate of the ISGMR average energies that depend on the nuclear densities, single-particle effects play a minor role on these energies even for drip line nuclei. In this regard, the macroscopic description of the finite nucleus incompressibility based on a leptodermous expansion is still valid and allows for a qualitative understanding of the behavior of the scaled estimates of the ISGMR average energies even near the drip lines.

The global behavior of the average energies of the ISGMR estimated through constrained calculations is, in general, similar to the one exhibited by the scaled energies. These average energies are in general smaller in drip line nuclei than in stable ones. The effect is much more pronounced for the constrained energies calculated near the neutron drip line, in contrast to those calculated in the scaling approximation. Near the neutron drip line, the constrained estimate of excitation energy of the ISGMR clearly deviates from the empirical $A^{-1 / 3}$ law known for stable nuclei. The reason for this behavior of the constrained estimate near the drip lines is that the single-particle effects are much more important in this case than in the scaling calculation. Nuclei near the drip lines are characterized by neutrons (protons) occupying very lightly bound levels whose corresponding wave functions extend very far from the core of the nucleus, especially in the case of neutron-rich nuclei owing to the absence of a Coulomb barrier. Neutrons occupying these orbits have larger single-particle neutron mean square radius and at the same time are much softer against pickup than those filling more bound orbits. Consequently, both the energy and the inverse energy-weighted sum rules are enhanced, this effect being more important in the latter than in the former. This explains the sizable decrease of the constrained estimate of the average energy of the ISGMR in nuclei near the neutron drip lines. This 
effect is particularly important in the $\mathrm{Ca}$ and $\mathrm{Zr}$ isotopic chains, where the corresponding neutron drip nuclei $\left({ }^{76} \mathrm{Ca}\right.$ and ${ }^{136} \mathrm{Zr}$, respectively) have the last occupied orbit bound by less than $1 \mathrm{MeV}$. Moreover for the particular nuclei ${ }^{52} \mathrm{Ca}$, and ${ }^{54} \mathrm{Ca}$, which are not neutron drip nuclei, we find a noticeable enhancement of the $m_{1}$ and $m_{-1}$ sum rules because for these specific nuclei, the neutron $2 p_{3 / 2}$ and $2 p_{1 / 2}$ single-particle wave functions extend very far from the core, although their binding energies are not particularly small.

The RPA sum rule description of some of the average properties of the ISGMR has some clear limitations as compared with a full RPA calculation of the response function. Thus only some global trends of the RPA strength can be inferred from our calculation. For instance, the reduction of the scaled and constrained energies near the drip lines should be associated with the appearance of a noticeable RPA strength in the low-energy region as suggested by the enhancement of the $m_{-1}$ sum rule. As in a full RPA calculation, nuclei near the drip lines are found to have a large resonance width in our approach, but the mean energy of the resonance cannot be determined very precisely because the scaled and constrained energies, which are upper and lower bounds of the mean energy, are largely separated. This large width near the drip lines can be due to a very broad resonance as well as to a fragmentation of the strength distribution.

We have also investigated the ability of semiclassical approximations of the TF type and its extensions including $\hbar$ corrections (ETF) for describing the ISGMR near the drip lines within the sum rule approach. The semiclassical sum rules are obtained by using the full quantal RPA expressions but with the HF expectation values replaced by the semiclassical ones at the TF or the ETF levels. The semiclassical estimates of the average scaled and constrained energies are free from any shell effects. Thus some of the discussed trends as, for instance, the enhancement of the $m_{1}$ and $m_{-1}$ sum rules when a particular orbital is occupied are only taken into account on the average in the semiclassical description. This means that the quantal RPA scaled and constrained energies may be spread around the values provided by the semiclassical calculations. In nuclei near the drip lines, the pure $\mathrm{TF}$ approximation fails in reproducing, even on the average, the global trends of the RPA constrained energies and thus the widths of the ISGMR, because of the poor description of the nuclear surface. However, when the $\hbar^{2}$-order corrections, and those of $\hbar^{4}$ order, are included in the semiclassical calculation, the ETF description of the surface of the nuclei agrees better with the HF one, and the RPA scaled and constrained energies are nicely averaged by the corresponding semiclassical counterpart.

We known that the right description of nuclei near the drip lines has to take into account pairing correlations, in particular for open shell nuclei. The inclusion of pairing in RPA, i.e., the quasiparticle RPA, has a noticeable effect on the strength of the response of the drip line nuclei to external fields [39]; however, its impact on the average excitation energies is much less. Thus, in the present approximation we did not consider pairing and assumed the uniform filling approach to obtain insight into the general trends of the ISGMR in exotic nuclei near the drip lines. In a next step, the application of the sum rule approach in the quasiparticle RPA should be probed to properly take into account the influence of pairing correlations on the average properties of the ISGMR. Investigations in this direction are in progress.

\section{ACKNOWLEDGMENTS}

Useful discussions with J. Martorell, G. Colò, N. Van Giai, and S. Shlomo are gratefully acknowledged. This work was partially supported by Grant Nos. BFM2002-01868 (DGI, Spain, and FEDER) and 2001SGR-00064 (DGR, Catalonia).
[1] Proceedings of the International Conference on Exotic Nuclei and Atomic Masses “ENAM 95”, Arles France, 1995, edited by M. de Saint Simon and O. Sorlin (Editions Frontiéres, Gif-surYvette, 1995).

[2] Proceedings of the International Workshop on the Physics and Techniques of Secondary Nuclear Beams, Dourdan, France, edited by J. F. Bruandet, B. Fernández, and M. Bex (Editions Frontiéres, Gif-sur-Yvette, 1992).

[3] Proceedings of the International Workshop on Research with Fission Fragments, Benediktbeuern, Germany, edited by T. Von Egidy et al. (World Scientific, Singapore, 1997).

[4] I. Tanihata, Heavy Ion Phys. 6, 143 (1997).

[5] T. Radon et al., Phys. Rev. Lett. 78, 4701 (1997).

[6] I. Hamamoto, H. Sagawa, and X. Z. Zhang, Phys. Rev. C 64, 024313 (2001).

[7] J. P. Blaizot, J. F. Berger, J. Dechargé, and M. Girod, Nucl. Phys. A591, 435 (1995).

[8] Nguyen Van Giai, P. F. Bortignon, G. Colò, Z.-Y. Ma, and M. R. Quaglia, Nucl. Phys. A687, 44c (2001).

[9] A. Bohr and B. R. Mottelson, Nuclear Structure (Benjamin, London, 1975), Vol. 2.
[10] G. F. Bertsch and S. F. Tsai, Phys. Rep. 18, 125 (1975).

[11] P. Ring and P. Schuck, The Nuclear Many-Body Problem (Springer-Verlag, Berlin, 1980).

[12] O. Bohigas, A. M. Lane, and J. Martorell, Phys. Rep. 51, 267 (1979).

[13] J. P. Blaizot, Phys. Rep. 64, 171 (1980).

[14] B. K. Jennings and A. D. Jackson, Phys. Rep. 66, 141 (1980).

[15] J. Treiner, H. Krivine, O. Bohigas, and J. Martorell, Nucl. Phys. A371, 253 (1981).

[16] I. Hamamoto, H. Sagawa, and X. Z. Zhang, Phys. Rev. C 53, 765 (1996).

[17] I. Hamamoto, H. Sagawa, and X. Z. Zhang, Phys. Rev. C 56, 3121 (1997).

[18] H. Sagawa, I. Hamamoto, and X. Z. Zhang, J. Phys. G 24, 1445 (1998).

[19] I. Hamamoto, H. Sagawa, and X. Z. Zhang, Phys. Rev. C 55, 2361 (1997).

[20] M. Brack, C. Guet, and H.-B. Håkansson, Phys. Rep. 123, 275 (1985).

[21] M. Brack and R. K. Bhaduri, Semiclassical Physics (AddisonWesley, Reading, 1997). 
[22] I. Zh. Petkov and M. V. Stoitsov, Nuclear Density Functional Theory (Clarendon Press, Oxford, 1991).

[23] H. A. Bethe and F. Bacher, Rev. Mod. Phys. 8, 82 (1936); C. F. von Weizsäcker, Z. Phys. 96, 431 (1935).

[24] P. Gleissl, M. Brack, J. Meyer, and P. Quentin, Ann. Phys. (NY) 197, 205 (1990).

[25] M. Centelles, M. Pi, X. Viñas, F. Garcias, and M. Barranco, Nucl. Phys. A510, 397 (1990).

[26] L. Guo-Quiang and X. Gong-Ou, Phys. Rev. C 42, 290 (1990).

[27] Li Guo-Quiang, J. Phys. G 17, 1 (1991).

[28] D. J. Thouless, Nucl. Phys. 22, 78 (1961).

[29] D. M. Brink and R. Leonardi, Nucl. Phys. A258, 285 (1976).

[30] H. Sagawa, Phys. Rev. C 65, 064314 (2002).

[31] B. K. Agrawal and S. Shlomo, Phys. Rev. C 70, 014308 (2004).
[32] F. Catara, C. H. Dasso, and A. Vitturi, Nucl. Phys. A602, 181 (1996).

[33] I. Hamamoto and H. Sagawa, Phys. Rev. C 53, R1492 (1996).

[34] M. Buenerd, D. Lebrun, Ph. Martin, P. de Saintignon, and C. Perrin, Phys. Rev. Lett. 45, 1667 (1980).

[35] U. Garg, P. Bogucki, J. D. Bronson, Y.-W. Lui, C. M. Rozsa, and D. H. Youngblood, Phys. Rev. Lett. 45, 1670 (1980).

[36] D. H. Youngblood, Y.-W. Lui, and H. L. Clark, Phys. Rev. C 60, 067302 (1999).

[37] M. Itoh et al., Phys. Lett. B549, 58 (2002).

[38] J. N. De, X. Viñas, S. K. Patra, and M. Centelles, Phys. Rev. C 64, 057306 (2001).

[39] M. Matsuo, Nucl. Phys. A696, 371 (2001); E. Khan, N. Sandulescu, M. Grasso, and N. V. Giai, Phys. Rev. C 66, 024309 (2002); N. Paar, P. Ring, T. Niksić, and D. Vretenar, Phys. Rev. C. 67, 034312 (2003). 